\title{
Fast Fourier Optimization
}

\section{Sparsity Matters}

\section{Robert J. Vanderbei}

Received: date / Accepted: date

\begin{abstract}
Many interesting and fundamentally practical optimization problems, ranging from optics, to signal processing, to radar and acoustics, involve constraints on the Fourier transform of a function. It is well-known that the fast Fourier transform (fft) is a recursive algorithm that can dramatically improve the efficiency for computing the discrete Fourier transform. However, because it is recursive, it is difficult to embed into a linear optimization problem. In this paper, we explain the main idea behind the fast Fourier transform and show how to adapt it in such a manner as to make it encodable as constraints in an optimization problem. We demonstrate a real-world problem from the field of high-contrast imaging. On this problem, dramatic improvements are translated to an ability to solve problems with a much finer grid of discretized points. As we shall show, in general, the "fast Fourier" version of the optimization constraints produces a larger but sparser constraint matrix and therefore one can think of the fast Fourier transform as a method of sparsifying the constraints in an optimization problem, which is usually a good thing.
\end{abstract}

Keywords Linear Programming · Fourier transform · interior-point methods · high-contrast imaging $\cdot \mathrm{fft} \cdot$ fast Fourier transform · optimization · Cooley-Tukey algorithm

Mathematics Subject Classification (2010) MSC 90C08 • 65T50 . $78 \mathrm{~A} 10$

The author was supported by a grant from NASA.

Robert J. Vanderbei

Department of Ops. Res. and Fin. Eng., Princeton University, Princeton, NJ 08544.

Tel.: +609-258-2345

E-mail: rvdb@princeton.edu 


\section{Fourier Transforms in Engineering}

Many problems in engineering involve maximizing (or minimizing) a linear functional of an unknown real-valued design function $f$ subject to constraints on its Fourier transform $\widehat{f}$ at certain points in transform space ([1]). Examples include antenna array synthesis (see, e.g., 12,13,16]), FIR filter design (see, e.g., , 4, 23, 24]), and coronagraph design (see, e.g., [8, 19, 11, 10, 17, 20,7, 9, 14]). If the design function $f$ can be constrained to vanish outside a compact interval $C=(-a, a)$ of the real line centered at the origin, then we can write the Fourier transform as

$$
\widehat{f}(\xi)=\int_{-a}^{a} e^{2 \pi i x \xi} f(x) d x
$$

and an optimization problem might look like

$$
\begin{array}{lll}
\text { maximize } & \int_{-a}^{a} c(x) f(x) d x & \\
\text { subject to } & -\varepsilon \leq \Re \widehat{f}(\xi) \leq \varepsilon, & \xi \in D \\
& -\varepsilon \leq \Im \widehat{f}(\xi) \leq \varepsilon, & \xi \in D \\
& 0 \leq f(x) \leq 1, & x \in C,
\end{array}
$$

where $D$ is a given subset of the real line, $\varepsilon$ is a given constant, and $\Re(z)$ and $\Im(z)$ denote the real and imaginary parts of the complex number $z$. In Section 7. we will discuss a specific real-world problem that fits a two-dimensional version of this optimization paradigm and for which dramatic computational improvements can be made.

Problem (1) is linear but it is infinite dimensional. The first step to making a tractable problem is to discretize both sets $C$ and $D$ so that the continuous Fourier transform can be approximated by a discrete Riemann sum:

$$
\widehat{f_{j}}=\sum_{k=-n}^{n} e^{2 \pi i k \Delta x j \Delta \xi} f_{k} \Delta x, \quad-n \leq j \leq n .
$$

Here, $n$ denotes the level of discretization,

$$
\Delta x=\frac{2 a}{2 n+1},
$$

$\Delta \xi$ denotes the discretization spacing in transform space, $f_{k}=f(k \Delta x)$, and $\widehat{f_{j}} \approx \widehat{f}(j \Delta \xi)$.

Computing the discrete approximation (2) by simply summing the terms in its definition requires on the order of $N^{2}$ operations, where $N=2 n+1$ is the number of discrete points in both the function space and the transform space (later we will generalize to allow a different number of points in the discretization of $C$ and $D$ ).

Choosing $\Delta \xi$ too large creates redundancy in the discrete approximation due to periodicity of the complex exponential function and hence one generally chooses $\Delta \xi$ such that

$$
\Delta x \Delta \xi \leq \frac{1}{N} .
$$


In many real-world applications, $\Delta \xi$ is chosen so that this inequality is an equality: $\Delta \xi=1 /(N \Delta x)$. In this case, the Riemann sum approximation is called the discrete Fourier transform.

\section{A Fast Fourier Transform}

Over the past half century there has been an explosion of research into algorithms for efficiently computing Fourier transforms. Any algorithm that can do the job in a constant times $N \log N$ multiplications/additions is called a fast Fourier transform (see, e.g., [5, 2, 15,6]). There are several algorithms that can be called fast Fourier transforms. Here, we present one that applies naturally to Fourier transforms expressed as in 22. In this section, we assume that $\Delta \xi=1 /(N \Delta x)$.

A sum from $-n$ to $n$ has an odd number of terms: $N=2 n+1$. Suppose, for this section, that $N$ is a power of three:

$$
N=3^{m} \text {. }
$$

Fast Fourier transform algorithms assume that it is possible to factor $N$ into a product

$$
N=N_{0} N_{1} \text {. }
$$

For the algorithm of this section, we put

$$
N_{0}=3, \quad \text { and } \quad N_{1}=3^{m-1} .
$$

The first key idea in fast Fourier transform algorithms is to write the single sum (1) as a double sum and simultaneously to represent the discrete set of transform values as a two-dimensional array of values rather than as a onedimensional vector. Specifically, we decompose $k$ as

$$
k=N_{0} k_{1}+k_{0}
$$

so that

$$
-n \leq k \leq n \quad \Longleftrightarrow \quad-n_{0} \leq k_{0} \leq n_{0} \quad \text { and } \quad-n_{1} \leq k_{1} \leq n_{1},
$$

where

$$
n_{0}=\left(N_{0}-1\right) / 2=(3-1) / 2=1
$$

and

$$
n_{1}=\left(N_{1}-1\right) / 2=\left(3^{m-1}-1\right) / 2 .
$$

Similarly, we decompose $j$ as

$$
j=N_{1} j_{1}+j_{0}
$$

so that

$$
-n \leq j \leq n \quad \Longleftrightarrow \quad-n_{1} \leq j_{0} \leq n_{1} \quad \text { and } \quad-1 \leq j_{1} \leq 1 .
$$


With these notations, we rewrite the Fourier transform (2) as a double sum:

$$
\widehat{f}_{j_{0}, j_{1}}=\sum_{k_{0}=-1}^{1} \sum_{k_{1}=-n_{1}}^{n_{1}} e^{2 \pi i\left(N_{0} k_{1}+k_{0}\right) \Delta x\left(N_{1} j_{1}+j_{0}\right) \Delta \xi} f_{k_{0}, k_{1}} \Delta x,
$$

where $f_{k_{0}, k_{1}}=f_{N_{0} k_{1}+k_{0}}$ and $\widehat{f}_{j_{0}, j_{1}}=\widehat{f}_{N_{1} j_{1}+j_{0}}$. Distributing the multiplications over the sums, we can rewrite the exponential as

$$
\begin{aligned}
& e^{2 \pi i\left(N_{0} k_{1}+k_{0}\right) \Delta x\left(N_{1} j_{1}+j_{0}\right) \Delta \xi} \\
& =e^{2 \pi i N_{0} k_{1} \Delta x\left(N_{1} j_{1}+j_{0}\right) \Delta \xi} e^{2 \pi i k_{0} \Delta x\left(N_{1} j_{1}+j_{0}\right) \Delta \xi} \\
& =e^{2 \pi i N_{0} k_{1} \Delta x N_{1} j_{1} \Delta \xi} e^{2 \pi i N_{0} k_{1} \Delta x j_{0} \Delta \xi} e^{2 \pi i k_{0} \Delta x\left(N_{1} j_{1}+j_{0}\right) \Delta \xi} \\
& =e^{2 \pi i N_{0} k_{1} \Delta x j_{0} \Delta \xi} e^{2 \pi i k_{0} \Delta x\left(N_{1} j_{1}+j_{0}\right) \Delta \xi},
\end{aligned}
$$

where the last equality follows from our assumption that $N_{0} N_{1} \Delta x \Delta \xi=N \Delta x \Delta \xi=$ 1. Substituting into (3), we get

$$
\widehat{f}_{j_{0}, j_{1}}=\sum_{k_{0}=-1}^{1} e^{2 \pi i k_{0} \Delta x\left(N_{1} j_{1}+j_{0}\right) \Delta \xi}\left(\sum_{k_{1}=-n_{1}}^{n_{1}} e^{2 \pi i N_{0} k_{1} \Delta x j_{0} \Delta \xi} \cdot f_{k_{0}, k_{1}}\right) \Delta x .
$$

We can compute this nested sum in two steps:

$$
\begin{array}{rlrl}
g_{j_{0}, k_{0}} & =\sum_{k_{1}=-n_{1}}^{n_{1}} e^{2 \pi i N_{0} k_{1} \Delta x j_{0} \Delta \xi} f_{k_{0}, k_{1}} \Delta x, & -n_{1} & \leq j_{0} \\
-1 & \leq n_{0} & \leq 1 \\
\widehat{f}_{j_{0}, j_{1}} & =\sum_{k_{0}=-1}^{1} e^{2 \pi i k_{0} \Delta x j \Delta \xi} g_{j_{0}, k_{0}}, & -n_{1} & \leq j_{0} \leq n_{1}, \\
-1 & \leq j_{1} \leq 1 .
\end{array}
$$

By design, computing $\widehat{f}_{j_{0}, j_{1}}$ for $-n_{1} \leq j_{0} \leq n_{1}$ and $-1 \leq j_{1} \leq 1$ is equivalent to computing $\widehat{f}_{j}$ for $-n \leq j \leq n$.

\subsection{Complexity}

If we compute $\widehat{f}_{j_{0}, j_{1}}$ in two steps according to the equations given above, then the number of multiply/adds is

$$
N_{1}^{2} N_{0}+N N_{0}=N\left(N_{1}+N_{0}\right) .
$$

On the other hand, the one-step algorithm given by $(2)$ requires $N^{2}$ multiply/adds. Hence, the two-step algorithm beats the one-step algorithm by a factor of

$$
\frac{N^{2}}{N\left(N_{1}+N_{0}\right)}=\frac{N}{N_{1}+N_{0}} \approx N / N_{1}=N_{0}=3 .
$$




\subsection{Recursive Application}

One can do better by iterating the above two-step algorithm. From the formula for $g_{j_{0}, k_{0}}$ given in (4), we see that $g$ is a discrete Fourier transform of a subset of the elements of the vector $\left\{f_{k}: k=-n, \ldots, n\right\}$ obtained by sampling $f$ at a cadence of one every $N_{0}$ elements. And, the coefficient $N_{0} \Delta x \Delta \xi$ in the exponential equals $N_{0} / N=1 / N_{1}$, which again matches the number of terms in the sum. Hence, we can apply the two-step algorithm again to this Fourier transform. The second key component of the fast Fourier transform is the observation that this process can be repeated until the Fourier transform only involves a sum consisting of a single term.

Let $I_{N}$ denote the number of multiply/adds needed using the recursive algorithm to solve a problem of size $N=3^{m}$. Keeping in mind that $N_{0}=3$, we get

$$
\begin{aligned}
I_{N}=I_{3^{m}} & =3 I_{3^{m-1}}+3 \cdot 3^{m} \\
& =3\left(3 I_{3^{m-2}}+3 \cdot 3^{m-1}\right)+3^{m+1} \\
& =3^{2} I_{3^{m-2}}+2 \cdot 3^{m+1} \\
& \vdots \\
& =3^{k} I_{3^{m-k}}+k \cdot 3^{m+1} \\
& \vdots \\
& =3^{m} I_{3^{0}}+m \cdot 3^{m+1} \\
& =3^{m}(1+3 m) \\
& =N\left(1+3 \log _{3} N\right) .
\end{aligned}
$$

Hence, the recursive variant of the algorithm takes on the order of $N \log _{3} N$ operations.

\section{A General Factor-Based Algorithm}

The advantage of fast Fourier transforms, such as the one presented in the previous section, is that they have order $N \log N$ complexity. But, they have disadvantages too. One disadvantage is the need to apply the basic two-step computation recursively. Recursion is fine for computing a Fourier transform, but our aim is to encode a Fourier transform within an optimization model. In such a context, it is far better to use a non-recursive algorithm.

A simple modification to the two-step process described in the previous section produces a variant of the two-step algorithm that makes a more substantial improvement in the initial two-step computation than what we obtained before. The idea is to factor $N$ into a pair of factors with each factor close to the square-root of $N$ rather than into 3 and $N / 3$. Indeed, in this section, we assume, as before, that $N$ can be factored into

$$
N=N_{0} N_{1}
$$


but we do not assume that $N_{0}=3$. In fact, we prefer to have $N_{0} \approx N_{1}$. As before, we assume that $N=2 n+1$ is odd and therefore that both $N_{0}$ and $N_{1}$ are odd:

$$
N_{0}=2 n_{0}+1 \quad \text { and } \quad N_{1}=2 n_{1}+1 .
$$

At the same time, we will now assume that the number of points in the discretization of the Fourier transform does not necessarily match the number of points in the discretization of the function itself. In many real-world examples, the "resolution" of the one discretization does not need to match the other and artificially enforcing such a match invariably results in a slower algorithm. So, suppose that the discrete Fourier transform has the form

$$
\widehat{f_{j}}=\sum_{k=-n}^{n} e^{2 \pi i k \Delta x j \Delta \xi} f_{k} \Delta x, \quad-m \leq j \leq m
$$

and let $M=2 m+1$ denote the number of elements in the discretized transform. Again, $M$ is odd and therefore we factor it into a product $M=M_{0} M_{1}$ of two odd factors:

$$
M_{0}=2 m_{0}+1 \quad \text { and } \quad M_{1}=2 m_{1}+1
$$

If we now decompose our sequencing indices $k$ and $j$ into

$$
k=N_{0} k_{1}+k_{0} \quad \text { and } \quad j=M_{0} j_{1}+j_{0}
$$

we get

$$
\begin{gathered}
\widehat{f} j_{0}, j_{1} \\
=\sum_{k_{0}=-n_{0}}^{n_{0}} \sum_{k_{1}=-n_{1}}^{n_{1}} e^{2 \pi i N_{0} k_{1} \Delta x M_{0} j_{1} \Delta \xi} e^{2 \pi i N_{0} k_{1} \Delta x j_{0} \Delta \xi} e^{2 \pi i k_{0} \Delta x\left(M_{0} j_{1}+j_{0}\right) \Delta \xi} \\
\cdot f_{k_{0}, k_{1}} \Delta x .
\end{gathered}
$$

As before, we need to assume that the first exponential factor evaluates to one. To make that happen, we assume that $N_{0} M_{0} \Delta x \Delta \xi$ is an integer. In realworld problems, there is generally substantial freedom in the choice of each of these four factors and therefore guaranteeing that the product is an integer is generally not a restriction. With that first exponential factor out of the way, we can again write down a two-step algorithm

$$
\begin{array}{rlrl}
g_{j_{0}, k_{0}} & =\sum_{k_{1}=-n_{1}}^{n_{1}} e^{2 \pi i N_{0} k_{1} \Delta x j_{0} \Delta \xi} f_{k_{0}, k_{1}} \Delta x, & & -m_{0} \leq j_{0} \leq m_{0}, \\
& -n_{0} \leq k_{0} \leq n_{0}, \\
\widehat{f}_{j_{0}, j_{1}}=\sum_{k_{0}=-n_{0}}^{n_{0}} e^{2 \pi i k_{0} \Delta x\left(M_{0} j_{1}+j_{0}\right) \Delta \xi} g_{j_{0}, k_{0},}, & & -m_{0} \leq j_{0} \leq m_{0} \\
& -m_{1} \leq j_{1} \leq m_{1} .
\end{array}
$$




\subsection{Complexity}

The number of multiply/adds required for this two-step algorithm is

$$
N M_{0}+M N_{0}=M N\left(\frac{1}{M_{1}}+\frac{1}{N_{1}}\right) .
$$

If $M \approx N$ and $M_{1} \approx N_{1} \approx \sqrt{N}$, the complexity simplifies to

$$
2 N \sqrt{N} \text {. }
$$

Compared to the one-step algorithm, which takes $N^{2}$ multiply/adds, this twostep algorithm gives an improvement of a factor of $\sqrt{N} / 2$. This first-iteration improvement is much better than the factor of 3 improvement from the first iteration of the recursive algorithm of the previous section. Also, if $M$ is much smaller than $N$, we get further improvement over the full $N \times N$ case.

Of course, if $M_{0}, M_{1}, N_{0}$, and $N_{1}$ can be further factored, then this twostep algorithm can be extended in the same manner as was employed for the algorithm of the previous section successively factoring $M$ and $N$ until it is reduced to prime factors. But, our eventual aim in this paper is to embed these algorithms into an optimization algorithm and so we will focus our attention in this paper just on two-step algorithms and not their recursive application.

\section{Fourier Transforms in 2D}

Many real-world optimization problems, and in particular the one to be discussed in Section 7, involve Fourier transforms in more than one dimension. It turns out that the core idea in the algorithms presented above, replacing a one-step computation with a two-step equivalent, presents itself in this higherdimensional context as well [18.

Consider a two-dimensional Fourier transform

$$
\widehat{f}(\xi, \eta)=\iint e^{2 \pi i(x \xi+y \eta)} f(x, y) d y d x
$$

and its discrete approximation

$$
\widehat{f}_{j_{1}, j_{2}}=\sum_{k_{1}=-n}^{n} \sum_{k_{2}=-n}^{n} e^{2 \pi i\left(x_{k_{1}} \xi_{j_{1}}+y_{k_{2}} \eta_{j_{2}}\right)} f_{k_{1}, k_{2}} \Delta y \Delta x, \quad-m \leq j_{1}, j_{2} \leq m,
$$

where

$$
\begin{aligned}
x_{k} & =k \Delta x, & & -n \leq k \leq n, \\
y_{k} & =k \Delta y, & & -n \leq k \leq n, \\
\xi_{j} & =j \Delta \xi, & & -m \leq j \leq m, \\
\eta_{j} & =j \Delta \eta, & & -m \leq j \leq m, \\
f_{k_{1}, k_{2}}= & f\left(x_{k_{1}}, y_{k_{2}}\right), & & -n \leq k_{1}, k_{2} \leq n \\
\widehat{f}_{j_{1}, j_{2}}= & \widehat{f}\left(\xi_{j_{1}}, \eta_{j_{2}}\right), & & -m \leq j_{1}, j_{2} \leq m .
\end{aligned}
$$


Performing the calculation in the obvious way requires $M^{2} N^{2}$ complex additions and a similar number of multiplies. However, we can factor the exponential into the product of two exponentials and break the process into two steps:

$$
\begin{aligned}
& g_{j_{1}, k_{2}}=\sum_{k_{1}=-n}^{n} e^{2 \pi i x_{k_{1}} \xi_{j_{1}}} f_{k_{1}, k_{2}} \Delta x, \quad-m \leq j_{1} \leq m,-n \leq k_{2} \leq n, \\
& \widehat{f}_{j_{1}, j_{2}}=\sum_{k_{2}=-n}^{n} e^{2 \pi i y_{k_{2}} \eta_{j_{2}}} g_{j_{1}, k_{2}} \Delta y, \quad-m \leq j_{1}, j_{2} \leq m
\end{aligned}
$$

It is clear that, in this context, the two-step approach is simply to break up the two-dimensional integral into a nested pair of one-dimensional integrals. Formulated this way, the calculation requires only $M N^{2}+M^{2} N$ complex additions (and a similar number of multiplications).

The real-world example we shall discuss shortly involves a two-dimensional Fourier transform. Given that the idea behind speeding up a one-dimensional Fourier transform is to reformulate it as a two-dimensional transform and then applying the two-step speed up trick of the two-dimensional transform, we shall for the rest of the paper restrict our attention to problems that are two dimensional.

\section{Exploiting Symmetry}

Before discussing real-world examples and associated computational results, it is helpful to make one more simplifying assumption. If we assume that $f$ is invariant under reflection about both the $x$ and $y$ axes, i.e., $f(-x, y)=f(x, y)$ and $f(x,-y)=f(x, y)$ for all $x$ and $y$, then the transform has this same symmetry and is in fact real-valued. In this case, it is simpler to use an even number of grid-points $(N=2 n$ and $M=2 m)$ rather than an odd number and write the straightforward algorithm for the two-dimensional discrete Fourier transform as

$$
\widehat{f}_{j_{1}, j_{2}}=4 \sum_{k_{1}=1}^{n} \sum_{k_{2}=1}^{n} \cos \left(2 \pi x_{k_{1}} \xi_{j_{1}}\right) \cos \left(2 \pi y_{k_{2}} \eta_{j_{2}}\right) f_{k_{1}, k_{2}} \Delta y \Delta x, \quad 1 \leq j_{1}, j_{2} \leq m
$$


where

$$
\begin{aligned}
x_{k} & =(k-1 / 2) \Delta x, & & 1 \leq k \leq n, \\
y_{k} & =(k-1 / 2) \Delta y, & & 1 \leq k \leq n, \\
\xi_{j} & =(j-1 / 2) \Delta \xi, & & 1 \leq j \leq m, \\
\eta_{j} & =(j-1 / 2) \Delta \eta, & & 1 \leq j \leq m, \\
f_{k_{1}, k_{2}} & =f\left(x_{k_{1}}, y_{k_{2}}\right), & & 1 \leq k_{1}, k_{2} \leq n \\
\widehat{f}_{j_{1}, j_{2}} & \approx \widehat{f}\left(\xi_{j_{1}}, \eta_{j_{2}}\right), & & 1 \leq j_{1}, j_{2} \leq m .
\end{aligned}
$$

The two-step algorithm then takes the following form:

$$
\begin{array}{ll}
g_{j_{1}, k_{2}}=2 \sum_{k_{1}=1}^{n} \cos \left(2 \pi x_{k_{1}} \xi_{j_{1}}\right) f_{k_{1}, k_{2}} \Delta x, & 1 \leq j_{1} \leq m, 1 \leq k_{2} \leq n, \\
\widehat{f}_{j_{1}, j_{2}}=2 \sum_{k_{2}=1}^{n} \cos \left(2 \pi y_{k_{2}} \eta_{j_{2}}\right) g_{j_{1}, k_{2}} \Delta y, & 1 \leq j_{1}, j_{2} \leq m,
\end{array}
$$

\subsection{Complexity}

The complexity of the straightforward one-step algorithm is $m^{2} n^{2}$ and the complexity of the two-step algorithm is $m n^{2}+m^{2} n$. Since $m=M / 2$ and $n=N / 2$, we see that by exploiting symmetry the straightforward algorithm gets speeded up by a factor of 16 and the two-step algorithm gets speeded up by a factor of 8 . But, the improvement is better than that as complex arithmetic has also been replaced by real arithmetic. One complex add is the same as two real adds and one complex multiply is equivalent to four real multiplies and two real adds. Hence, complex arithmetic is about four times more computationally expensive than real arithmetic.

\section{Matrix Notation}

As Fourier transforms are linear operators it is instructive to express our algorithms in matrix/vector notation. In this section, we shall do this for the two-dimensional Fourier transform. To this end, let $F$ denote the $n \times n$ matrix with elements $f_{k_{1}, k_{2}}$, let $G$ denote the $m \times n$ matrix with elements $g_{j_{1}, k_{2}}$, let $\widehat{F}$ denote the $m \times m$ matrix with elements $\widehat{f}_{j_{1}, j_{2}}$, and let $K$ denote the $m \times n$ Fourier kernel matrix whose elements are

$$
\kappa_{j_{1}, k_{2}}=\cos \left(2 \pi x_{k_{1}} \xi_{j_{1}}\right) \Delta x
$$

For notational simplicity, assume that the discretization in $y$ is the same as it is in $x$, i.e., $\Delta x=\Delta y$, and that the discretization in $\eta$ is the same as it is in $\xi$, 
i.e., $\Delta \eta=\Delta \xi$. Then, the two-dimensional Fourier transform $\widehat{F}$ can be written simply as

$$
\widehat{F}=K F K^{T}
$$

and the computation of the transform in two steps is just the statement that the two matrix multiplications can, and should, be done separately:

$$
\begin{aligned}
& G=K F \\
& \widehat{F}=G K^{T} .
\end{aligned}
$$

When linear expressions are passed to a linear programming code, the variables are passed as a vector and the constraints are expressed in terms of a matrix of coefficients times this vector. The matrix $F$ above represents the variables in the optimization problem. If we let $f_{k}, k=1, \ldots, n$ denote the $n$ columns of this matrix, i.e., $F=\left[f_{1} f_{2} \cdots f_{n}\right]$, then we can list the elements in column-by-column order to make a column vector (of length $n^{2}$ ):

$$
\operatorname{vec}(F)=\left[\begin{array}{c}
f_{1} \\
f_{2} \\
\vdots \\
f_{n}
\end{array}\right] .
$$

Similarly, we can list the elements of $G$ and $\widehat{F}$ in column vectors too:

$$
\operatorname{vec}(G)=\left[\begin{array}{c}
g_{1} \\
g_{2} \\
\vdots \\
g_{n}
\end{array}\right] \quad \text { and } \quad \operatorname{vec}(\widehat{F})=\left[\begin{array}{c}
\widehat{f_{1}} \\
\widehat{f}_{2} \\
\vdots \\
\widehat{f}_{m}
\end{array}\right] \text {. }
$$

It is straightforward to check that

$$
\operatorname{vec}(G)=\left[\begin{array}{cccc}
K & & & \\
& K & & \\
& & \ddots & \\
& & & K
\end{array}\right] \operatorname{vec}(F)
$$

and that

$$
\operatorname{vec}(\widehat{F})=\left[\begin{array}{cccc}
\kappa_{1,1} I & \kappa_{1,2} I & \cdots & \kappa_{1, n} I \\
\kappa_{2,1} I & \kappa_{2,2} I & \cdots & \kappa_{2, n} I \\
\vdots & \vdots & \ddots & \vdots \\
\kappa_{m, 1} I & \kappa_{m, 2} I & \cdots & \kappa_{m, n} I
\end{array}\right] \operatorname{vec}(G)
$$

where $I$ denotes an $m \times m$ identity matrix.

The matrices in these two formulae are sparse: the first is block diagonal and the second is built from identity matrices. Passing the constraints to a solver as these two sets of constraints introduces new variables and more constraints, but the constraints are very sparse. Alternatively, if we were to 
express $\operatorname{vec}(\widehat{F})$ directly in terms of $\operatorname{vec}(F)$, these two sparse matrices would be multiplied together and a dense coefficient matrix would be passed to the solver. It is often the case that optimization problems expressed in terms of sparse matrices solve much faster than equivalent formulations involving dense matrices even when the latter involves fewer variables and/or constraints (see, e.g., 21]).

\section{A Real-World Example: High-Contrast Imaging}

Given the large number of planets discovered over the past decade by socalled "indirect" detection methods, there is great interest in building a special purpose telescope capable of imaging a very faint planet very close to its much brighter host star. This is a problem in high-contrast imaging. It is made difficult by the fact that light is a wave and therefore point sources, like the star and the much fainter planet, produce not just single points of light in the image but rather diffraction patterns - most of the light lands where ray-optics suggests it will but some of the light lands nearby but not exactly at this point. In a conventional telescope, the "wings" of the diffraction pattern produced by the star are many orders of magnitude brighter than any planet would be at the place where the planet might be. Hence, the starlight outshines the planet and makes the planet impossible to detect. But, it is possible to customize the diffraction pattern by designing an appropriate filter, or a mask, to put on the front of the telescope. While it is impossible to concentrate all of the starlight at the central point - to do so would violate the uncertainty principle - it is possible to control it in such a way that there is a very dark patch very close to the central spot.

Suppose that we place a filter over the opening of a telescope with the property that the transmissivity of the filter varies from place to place over the surface of the filter. Let $f(x, y)$ denote the transmissivity at location $(x, y)$ on the surface of the filter $((0,0)$ denotes the center of the filter $)$. It turns out that the electromagnetic field in the image plane of such a telescope associated with a single point on-axis source (the star) is proportional to the Fourier transform of the filter function $f$. Choosing units in such a way that the telescope's opening has a diameter of one, the Fourier transform can be written as

$$
\widehat{f}(\xi, \eta)=\int_{-1 / 2}^{1 / 2} \int_{-1 / 2}^{1 / 2} e^{2 \pi i(x \xi+y \eta)} f(x, y) d y d x .
$$

The intensity of the light in the image is proportional to the magnitude squared of the electromagnetic field.

Assuming that the underlying telescope has a circular opening of diameter one, we impose the following constraint on the function $f$ :

$$
f(x, y)=0 \quad \text { for } \quad x^{2}+y^{2}>(1 / 2)^{2} .
$$

As often happens in real-world problems, there are multiple competing goals. We wish to maximize the amount of light that passes through the filter 
and at the same time minimize the amount of light that lands within a dark zone $\mathcal{D}$ of the image plane. If too much light lands in the dark zone, the telescope will fail to detect the planets it is designed to find. Hence, this latter objective is usually formulated as a constraint. This leads to the following optimization problem:

$$
\begin{array}{ll}
\text { maximize } & \iint f(x, y) d y d x \\
\text { subject to } \quad|\widehat{f}(\xi, \eta)|^{2} \leq \varepsilon, & (\xi, \eta) \in \mathcal{D}, \\
& f(x, y)=0, \quad x^{2}+y^{2}>(1 / 2)^{2}, \\
0 \leq f(x, y) \leq 1, & \text { for all } x, y .
\end{array}
$$

Here, $\varepsilon$ is a small positive constant representing the maximum level of brightness of the starlight in the dark zone. Without imposing further symmetry constraints on the function $f$, the Fourier transform $\widehat{f}$ is complex valued. Hence this optimization problem has a linear objective function and both linear constraints and convex quadratic inequality constraints. Hence, a discretized version can be solved (to a global optimum) using, say, interior-point methods.

Assuming that the filter can be symmetric with respect to reflection about both axes (in real-world examples, this is often - but not always - possible; see [3] for several examples), the Fourier transform can be written as

$$
\widehat{f}(\xi, \eta)=4 \int_{0}^{1 / 2} \int_{0}^{1 / 2} \cos (2 \pi x \xi) \cos (2 \pi y \eta) f(x, y) d y d x .
$$

In this case, the Fourier transform is real and so the convex quadratic inequality constraint in (8) can be replaced with a pair of inequalities,

$$
-\sqrt{\varepsilon} \leq \widehat{f}(\xi, \eta) \leq \sqrt{\varepsilon}
$$

making the problem an infinite dimensional linear programming problem.

Figure 1 shows an AMPL model formulation of this problem expressed in the straightforward one-step manner. Figure 2, on the other hand, shows an AMPL model for the same problem but with the Fourier transform expressed as a pair of transforms - the so-called two-step process.

As Figures 3 , 4 and 5 show, the optimal solution for the two models are, of course, essentially the same except for the improved resolution in the two-step version provided by a larger value for $n(n=1000$ vs. $n=150)$. Using LOQO 22] as the interior-point method to solve the problems, both versions solve in a few hours on a modern computer. It is possible to solve even larger instances, say $n=2000$, if one is willing to wait a day or so for a solution. Ultimately, higher resolution is actually important because manufacturing these masks involves replacing the pixellated mask with a spline-fitted smooth approximation and it is important to get this approximation correct.

Table 1 summarizes problem statistics for the two versions of the model as well as a few other size choices. Table 2 summarizes solution statistics for these 


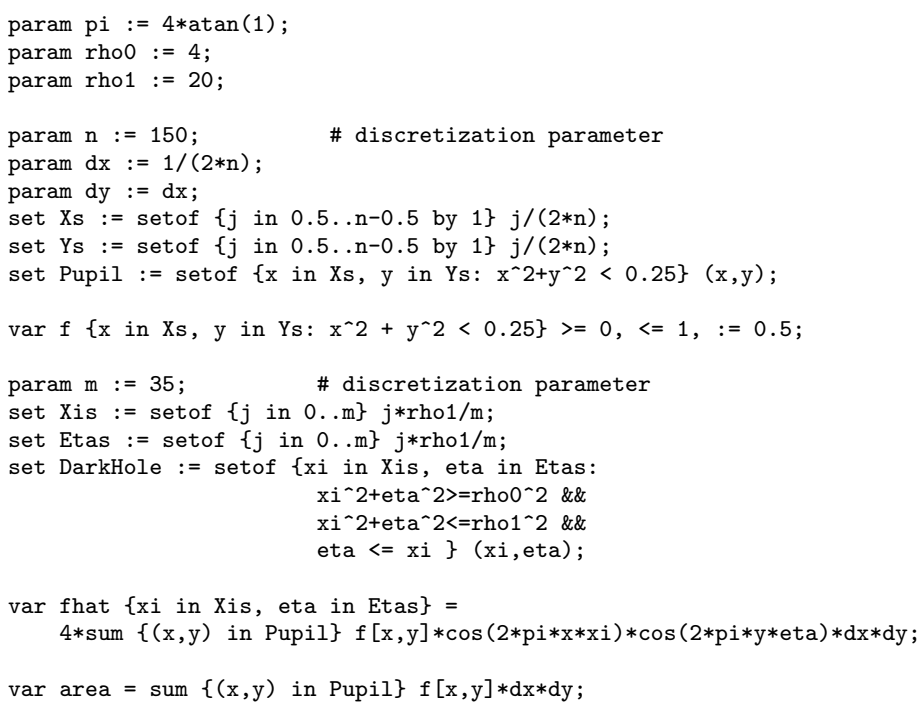

subject to sidelobe_pos $\{(\mathrm{xi}, \mathrm{eta})$ in DarkHole $\}$ : fhat $[\mathrm{xi}, \mathrm{eta}]<=10^{-}(-5) *$ fhat $[0,0]$; subject to sidelobe_neg $\{(\mathrm{xi}, \mathrm{eta})$ in DarkHole $\}$ : $-10^{-}(-5) *$ fhat $[0,0]<=$ fhat $[\mathrm{xi}, \mathrm{eta}]$;

solve;

Fig. 1 AMPL model for discretized version of problem 8 assuming that the mask is symmetric about the $x$ and $y$ axes. The dark zone $\mathcal{D}$ is a pair of sectors of an annulus with inner radius 4 and outer radius 20. The optimal solution is shown in Figure 3

Table 1 Comparison between a few sizes of the one-step model shown in Figure 1 and a few sizes of the two-step model shown in Figure 2 The column labeled nonzeros reports the number of nonzeros in the constraint matrix of the linear programming problem and the column arith. ops. The One-Step-250x35 problem is too large to solve by LOQO, which is compiled for a 32-bit architecture operating system.

\begin{tabular}{lrr|rrrr}
\hline Model & $n$ & $m$ & constraints & variables & nonzeros & arith. ops. \\
\hline One step & 150 & 35 & 976 & 17,672 & $17,247,872$ & $17,196,541,336$ \\
One step & 250 & 35 & $*$ & $*$ & $*$ & $*$ \\
\hline Two step & 150 & 35 & 7,672 & 24,368 & 839,240 & $3,972,909,664$ \\
Two step & 500 & 35 & 20,272 & 215,660 & $7,738,352$ & $11,854,305,444$ \\
Two step & 1000 & 35 & 38,272 & 822,715 & $29,610,332$ & $23,532,807,719$ \\
\hline
\end{tabular}

same problems. These problems were run as a single thread on a GNU/Linux (Red Hat Enterprise Linux Server release 5.7) x86_64 server with dual Xeon X5460s cpus (3.16 GHz with 4 cores each), $32 \mathrm{~GB}$ of RAM and a $6.1 \mathrm{MB}$ cache.

Real telescopes have opennings that are generally not just open unobstructed disks but, rather, typically have central obstructions supported by 
param pi := 4*atan $(1)$

param $r h o 0:=4$

param $r h o 1:=20$

param $\mathrm{n}:=1000$

param $\mathrm{dx}:=1 /(2 * \mathrm{n})$

param $d y:=d x$

set $X s:=$ setof $\{j$ in $0.5 \ldots n-0.5$ by 1$\} j /(2 * n)$;

set $Y_{s}:=$ setof $\{j$ in $0.5 \ldots n-0.5$ by 1$\} j /(2 * n)$;

set Pupil := setof $\left\{\mathrm{x}\right.$ in Xs, $\mathrm{y}$ in Ys: $\left.\mathrm{x}^{\wedge} 2+\mathrm{y}^{\wedge} 2<0.25\right\}(\mathrm{x}, \mathrm{y})$;

$\operatorname{var} \mathrm{f}\left\{\mathrm{x}\right.$ in $\mathrm{Xs}, \mathrm{y}$ in Ys: $\left.\mathrm{x}^{\wedge} 2+\mathrm{y}^{\wedge} 2<0.25\right\}>=0,<=1,:=0.5$

param $m:=35 ; \quad$ \# discretization parameter

set $X$ is $:=$ setof $\{j$ in $0 \ldots m\} j * r h o 1 / m$;

set Etas $:=$ setof $\{j$ in $0 \ldots m\} \quad j * r h o 1 / m$;

set DarkHole := setof $\{\mathrm{xi}$ in Xis, eta in Etas:

$$
\begin{aligned}
& x i^{\wedge} 2+\text { eta`2 }=r h o 0^{\wedge} 2 \text { \&\& } \\
& x i^{\wedge} 2+\text { eta`2<=rho1^2 \&\& } \\
& \text { eta }<=x i\} \text { (xi,eta); }
\end{aligned}
$$

$\operatorname{var} \mathrm{g}\{\mathrm{xi}$ in $\mathrm{Xis}, \mathrm{y}$ in $\mathrm{Ys}\}$;

var fhat $\{\mathrm{xi}$ in $\mathrm{Xis}$, eta in Etas\}.

var $\operatorname{area}=\operatorname{sum}\{(\mathrm{x}, \mathrm{y})$ in Pupil $\} \mathrm{f}[\mathrm{x}, \mathrm{y}] * \mathrm{dx} * \mathrm{dy}$;

maximize throughput: area;

subject to g_def $\{\mathrm{xi}$ in $\mathrm{Xis,} \mathrm{y}$ in $\mathrm{Ys}\}$ :

$\mathrm{g}[\mathrm{xi}, \mathrm{y}]=2 * \operatorname{sum}\{\mathrm{x}$ in $\mathrm{Xs}:(\mathrm{x}, \mathrm{y})$ in Pupil $\}$

$f[\mathrm{x}, \mathrm{y}] * \cos (2 * \mathrm{pi} * \mathrm{x} * \mathrm{xi}) * \mathrm{dx}$

subject to fhat_def $\{\mathrm{xi}$ in $\mathrm{Xis}$, eta in Etas $\}$

fhat $[\mathrm{xi}, \mathrm{eta}]=2 * \operatorname{sum}\{\mathrm{y}$ in Ys

$\mathrm{g}[\mathrm{xi}, \mathrm{y}] * \cos (2 *$ pi*y*eta $) *$ dy;

subject to sidelobe_pos $\{(\mathrm{xi}, \mathrm{eta})$ in DarkHole $\}$ : fhat $[\mathrm{xi}, \mathrm{eta}]<=10^{\wedge}(-5) *$ fhat $[0,0]$;

subject to sidelobe_neg $\{(\mathrm{xi}, \mathrm{eta})$ in DarkHole $\}$ : $-10^{-}(-5) *$ fhat $[0,0]<=$ fhat $[\mathrm{xi}$, eta];

solve;

Fig. 2 AMPL model reformulated to exploit the two-step algorithm. The optimal solution is shown in Figure 4

Table 2 Hardware-specific performance comparison data. The results shown here were obtained using the default value for all of LOQO's tunable parameters. It is possible to reduce the iteration counts to about 100 or less on all the problems by increasing the value of the epsdiag parameter to about $1 \mathrm{e}-9$

\begin{tabular}{lrr|rrrr}
\hline Model & $n$ & $m$ & iterations & primal objective & dual objective & cpu time (sec) \\
\hline One step & 150 & 35 & 54 & 0.05374227247 & 0.05374228041 & $*$ \\
One step & 250 & 35 & $*$ & $*$ & $*$ & $*$ \\
\hline Two step & 150 & 35 & 185 & 0.05374233071 & 0.05374236091 & 1064 \\
Two step & 500 & 35 & 187 & 0.05395622255 & 0.05395623990 & 4922 \\
Two step & 1000 & 35 & 444 & 0.05394366337 & 0.05394369256 & 26060 \\
\hline
\end{tabular}



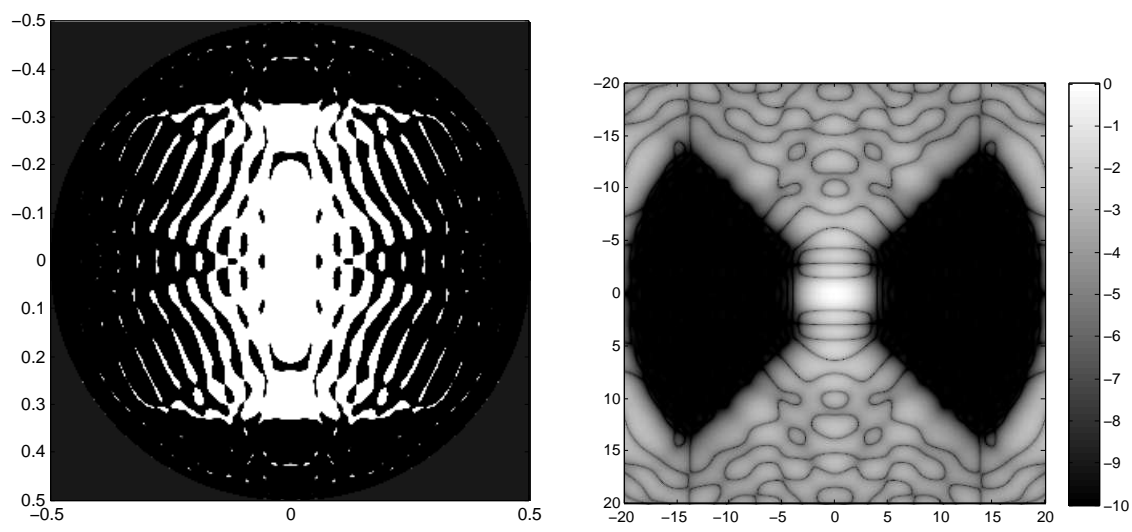

Fig. 3 The optimal filter from the one-step model shown in Figure 1 which turns out to be purely opaque and transparent (i.e., a mask), and a logarithmic plot of the star's image.
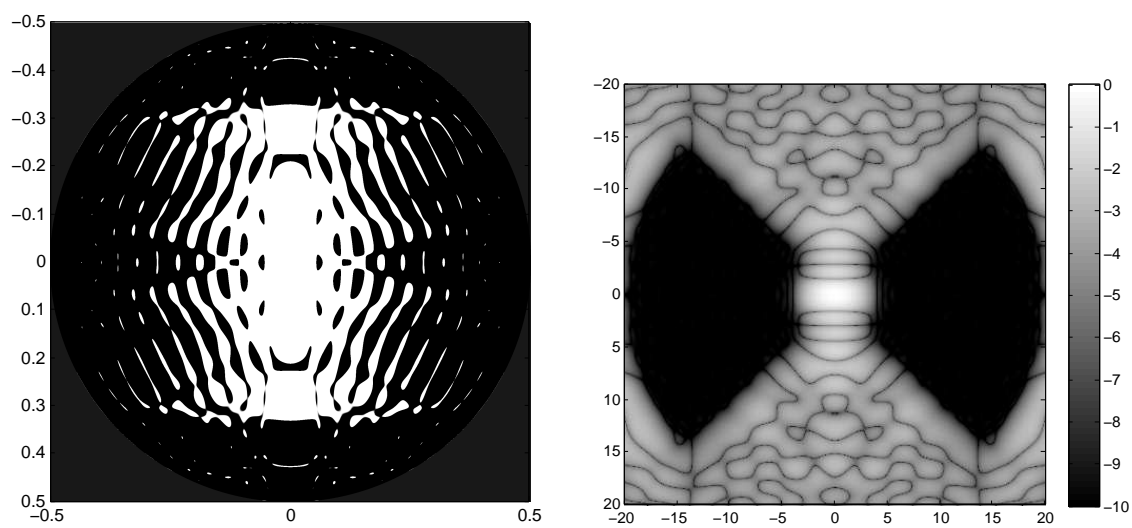

Fig. 4 The optimal filter from the two-step model shown in Figure 2 and a logarithmic plot of the star's image.

spiders. It is easy to extend the ideas presented here to handle such situations; see $[3$.

As explained in earlier sections, the two-step algorithm applied to a onedimensional Fourier transform effectively makes a two-dimensional representation of the problem and applies the same two-step algorithm that we have used for two-dimensional Fourier transforms. It is natural, therefore to consider whether we can get more efficiency gains by applying the two-step algorithm to each of the iterated one-dimensional Fourier transforms that make up the two-step algorithm for the two-dimensional Fourier transform. We leave such investigations for future work. 

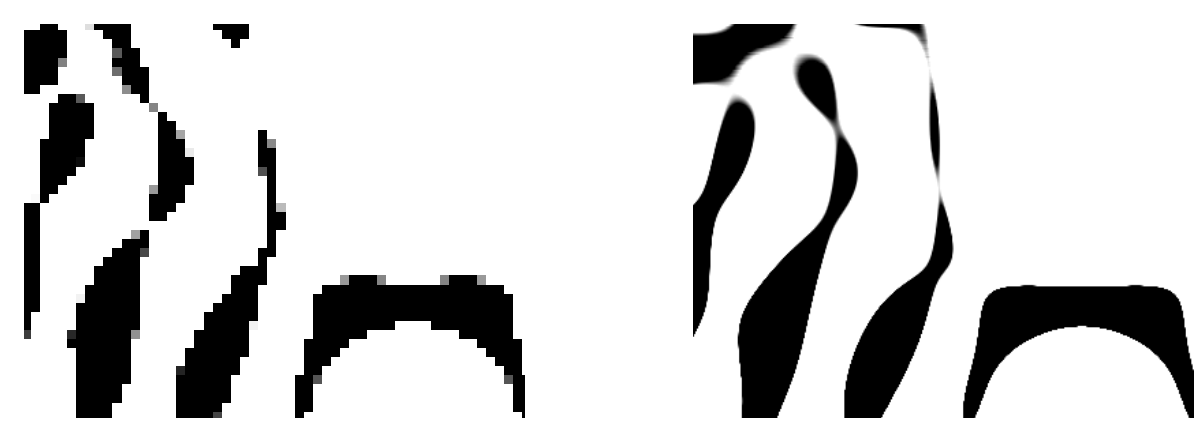

Fig. 5 Close up of the two masks to compare resolution.
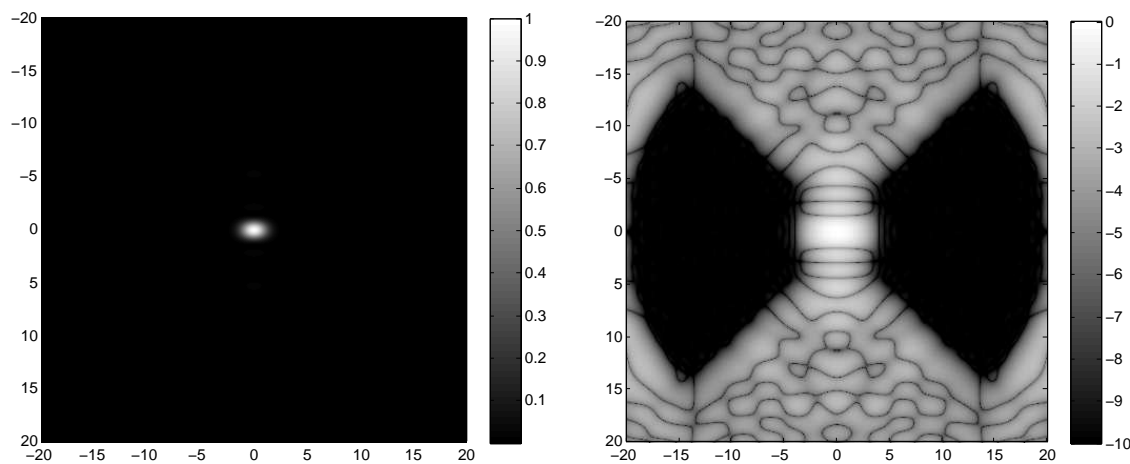

Fig. 6 Logarithmic stretches are useful but can be misleading. Left: the image of the star shown in a linear stretch. Right: the same image shown in a logarithmic stretch.

Acknowledgements I would like to thank N. Jeremy Kasdin, Alexis Carlotti, and all the members of the Princeton High-Contrast Imaging Lab for many stimulating discussions.

\section{References}

1. Ben-Tal, A., Nemirovsky, A.: Lectures on Modern Convex Optimization: Analysis, Algorithms, and Engineering Applications. SIAM (2001)

2. Brigham, E., Morrow, R.: The fast fourier transform. IEEE Spectrum 4(12), 63-70 (1967)

3. Carlotti, A., Vanderbei, R.J., Kasdin, N.J.: Optimal pupil apodizations for arbitrary apertures. Optics Express 19(27), 26,796-26,809 (2011)

4. Coleman, J., Scholnik, D.: Design of nonlinear-phase FIR filters with second-order cone programming. In: Proceedings of 1999 Midwest Symposium on Circuits and Systems (1999)

5. Cooley, J., Tukey, J.: An algorithm for the machine calculation of complex fourier series. Math. of Computation 19, 297-301 (1965)

6. Duhamel, P., Vetterli, M.: Fast fourier transforms: A tutorial review and a state of the art. Signal Processing 19, 259-299 (1990)

7. Guyon, O., Pluzhnik, E., Kuchner, M., Collins, B., Ridgway, S.: Theoretical limits on extrasolar terrestrial planet detection with coronagraphs. The Astrophysical Journal Supplement 167(1), 81-99 (2006) 
8. Indebetouw, G.: Optimal apodizing properties of gaussian pupils. Journal of Modern Optics 37(7), 1271-1275 (1990)

9. J.T.Trauger, Traub, W.: A laboratory demonstration of the capability to image an earth-like extrasolar planet. Nature 446(7137), 771-774 (2007)

10. Kasdin, N., Vanderbei, R., Littman, M., Spergel, D.: Optimal one-dimensional apodizations and shaped pupils for planet finding coronagraphy. Applied Optics 44(7), 1117$1128(2005)$

11. Kasdin, N., Vanderbei, R., Spergel, D., Littman, M.: Extrasolar Planet Finding via Optimal Apodized and Shaped Pupil Coronagraphs Astrophysical Journal 582, 1147$1161(2003)$

12. Lebret, H., Boyd, S.: Antenna array pattern synthesis via convex optimization. IEEE Transactions on Signal Processing 45, 526-532 (1997)

13. Mailloux, R.: Phased Array Antenna Handbook. Artech House (2005)

14. Martinez, P., Dorrer, C., Carpentier, E.A., Kasper, M., Boccaletti, A., Dohlen, K., Yaitskova, N.: Design, analysis, and testing of a microdot apodizer for the apodized pupil Lyot coronagraph. Astronomy and Astrophysics 495(1), 363-370 (2009)

15. Papadimitriou, C.: Optimality of the fast fourier transform. J. ACM 26, 95-102 (1979)

16. Scholnik, D., Coleman, J.: Optimal array-pattern synthesis for wideband digital transmit arrays. IEEE J. of Signal Processing 1(4), 660-677 (2007)

17. Soummer, R.: Apodized pupil lyot coronagraphs for arbitrary telescope apertures. The Astrophysical Journal Letters 618, 161-164 (2005)

18. Soummer, R., Pueyo, L., Sivaramakrishnan, A., Vanderbei, R.: Fast computation of lyot-style coronagraph propagation. Optics Express 15(24), 15,935-15,951 (2007)

19. Spergel, D., Kasdin, N.: A shaped pupil coronagraph: A simpler path towards TPF. Bulletin of the Amer. Astronomical Society 33, 1431 (2001)

20. Tanaka, S., Enya, K., L.Abe, Nakagawa, T., Kataza, H.: Binary-shaped pupil coronagraphs for high-contrast imaging using a space telescope with central obstructions. Publications of the Astronomical Society of Japan 58(3), 627-639 (2006)

21. Vanderbei, R.: Splitting dense columns in sparse linear systems. Lin. Alg. and Appl. 152, 107-117 (1991)

22. Vanderbei, R.: LOQO: An interior point code for quadratic programming. Optimization Methods and Software 12, 451-484 (1999)

23. Wu, S., Boyd, S., Vandenberghe, L.: FIR filter design via semidefinite programming and spectral factorization. Proc. of the IEEE Conf. on Decision and Control 35, 271-276 (1996)

24. Wu, S., Boyd, S., Vandenberghe, L.: FIR filter design via spectral factorization and convex optimization. In: B. Datta (ed.) Applied and Computational Control, Signals, and Circuits, vol. 1, pp. 215-246. Birkhauser (1999) 